\title{
SOBREDENTADURAS IMPLANTOASISTIDAS EN EL PACIENTE DESDENTADO TOTAL: BASES PARA SU INDICACIÓN
}

Implant-assisted overdentures in the totally edentulous patient: Basis for its indication

Fecha de Recepción: 21 de abril 2021
Sobredentaduras assistidas por implante no paciente totalmente edêntulo: Base para sua indicação

Aceptado para su publicación: 30 de julio 2021
Luis Guillermo Caputo ${ }^{1, a}$

Damian Luis De Moliner ${ }^{1, b}$

José Daniel Maquieira ${ }^{1, c}$

Pamela Boragina ${ }^{1, c}$

Jorge Esteban Aredes ${ }^{1, d}$

1. Cátedra Clínica 1 de Prótesis. Facultad de Odontología. Universidad de

Buenos Aires. República Argentina.

a. Especialista en Prótesis Dental (Universidad de

Buenos Aires)

b. Especialista en Prostodoncia (Universidad de

Buenos Aires)

c. Odontólogo/a (Universidad de Buenos Aires)

d. Doctor de la Universidad de Buenos Aires.

Área Odontología Rehabilitadora

Correspondencia:

Aredes Jorge Esteban

Cátedra Clínica 1 de Prótesis

UBA - Facultad de Odontología

Marcelo T. de Alvear 2142

+ 541152876000

Correo electrónico:

jorge.aredes@odontologia.uba.ar

Conflicto de intereses:

los autores declaran no tener conflictos de interés.

Fuente de financiamiento:

autofinanciado.

\section{Resumen}

El aumento de la edad de la población y el número relevante de pacientes edéntulos obligan a pensar su tratamiento como una necesidad actual y progresiva. El uso de implantes dentales deviene en alternativas de tratamiento de probada eficacia. Las sobredentaduras como nueva alternativa terapéutica están indicadas siempre en la mandíbula, mientras en el maxilar superior solo si existen piezas dentarias viables en el maxilar inferior. Se buscó literatura relacionada con el tema planteado, para desarrollar esta revisión narrativa, con el fin de estudiar las bases que indican las sobredentaduras implantoasistidas en el paciente desdentado total. La literatura científica internacional sugiere que el tratamiento de un desdentado total con prótesis completa convencional inferior no es actualmente el tratamiento más adecuado. Existe evidencia científica significativa que soporta la elección a priori de una sobredentadura inferior soportada por dos implantes, es vista como primera opción de tratamiento en un desdentado total.

Palabras clave: dentadura completa; sobredentadura; arcada edéntula (fuente: DeCS BIREME).

\section{Abstract}

The increase in the age of the population and the significant number of edentulous patients make it necessary to consider their treatment as a current and progressive need. The use of dental implants evolves into alternatives of treatment with proven efficacy. Overdentures as a new therapeutic alternative are always indicated in the mandible, while in the maxilla only if there are viable teeth in the mandible. Literature related to this topic was sought - to develop this narrative review - to study the bases that indicate implant-assisted overdentures in totally edentulous patients. The international scientific literature suggests that treatment of a totally edentu- 
Revista de la Facultad de Odontología

Sobredentaduras implantoasistidas en el paciente desdentado total: bases para su indicación.
REFO

VOL. XIV | N 2| AÑO 2021

ISSN 1668-7280

ISSN-E 2683-7986 lous patient with a conventional full lower denture is not currently the most appropriate treatment. There is significant scientific evidence to support the a priori choice of a lower overdenture supported by two implants as the first treatment option in a totally edentulous patient.

Key words: denture, complete; overdentures; jaws, edentulous (source: MeSH NLM).

\section{Resumo}

O aumento da idade da população e o número relevante de pacientes desdentados tornam necessário pensar o seu tratamento como uma necessidade atual e progressiva. O uso de implantes dentários torna-se uma alternativa de tratamento comprovadamente eficaz. As sobredentaduras como nova alternativa terapêutica são sempre indicadas na mandíbula, enquanto na maxila apenas se houver dentes viáveis na mandíbula. Buscou-se literatura relacionada ao tema levantado para desenvolver esta revisão narrativa, a fim de estudar as bases que indicam sobredentaduras assistidas por implante em pacientes desdentados totais. A literatura científica internacional sugere que o tratamento de um desdentado total com prótese total inferior convencional não é atualmente o tratamento mais adequado. Existem evidências científicas significativas que suportam a escolha a priori de uma sobredentadura inferior suportada por dois implantes, sendo vista como a primeira opção de tratamento em um edêntulo total.

Palavras-chave: prótese total; prótese híbrida; arcada edêntula (fonte: DeCS BIREME).

\section{Introducción}

El edentulismo es una situación clínica que, aun ingresados en el siglo XXI, no parece estar en extinción como se suponía. Las estadísticas detectan un aumento considerable de la perdida de dientes, junto con un progresivo envejecimiento de la población tanto en las sociedades de los países centrales como en los periféricos y en vías de desarrollo. En 2016, el Consejo de Dentistas Europeos (CED) recomendó la inclusión de la Gerodontología a la Comisión Europea, como parte de la revisión obligatoria del programa de estudios para odontólogos en Europa ${ }^{1}$.

El edentulismo es una condición devastadora e irreversible y se describe como el marcador final de la carga de morbilidad para la salud bucal ${ }^{2}$. La Encuesta Mundial sobre Salud de la OMS, realizada entre 2002 y 2004, demostró que es una condición altamente prevalente en todo el mundo. Para las personas de 65 años y mayores, había una prevalencia promedio general de $32,9 \%$ en países individuales, desde un $7 \%$ en Egipto hasta un $72 \%$ en Islandia 3 .

A su vez, es la primera vez en la historia de la humanidad que un gran número de personas llegan a la ancianidad ${ }^{4}$. Es evidente que todo este proceso ha provocado un determinado impacto en ciertos sectores estratégicos de la estructura social, entre los que se encuentran el sistema educacional y de salud $^{5}$. Ante esta situación, la $\mathrm{OMS}^{6}$ ha reconocido la necesidad de propiciar una formación adecuada a todo personal sanitario para que tengan las capacidades específicas que requiere atender a los adultos mayores ${ }^{7}$. Esta organización, está llamada a marcar mundialmente la pauta en la dimensión de los problemas sanitarios del envejecimiento, que ha pasado a ser una cuestión social de primera importancia en el siglo XXI, según sus informes y publicaciones ${ }^{8,9}$. Según las proyecciones más recientes de las Naciones Unidas, se espera que la cantidad de personas de 60 años o más en la región de Latinoamérica y el Caribe (LAC) aumente de 59 millones en 2010 a 196 millones en 2050 y la cantidad de personas de 80 años o más aumente de 8.6 millones a más de 44 millones durante el mismo periodo ${ }^{10,11}$. Las tasas de crecimiento de esta magnitud generarán una dinámica de población sin precedentes en la historia humana, sumado a un aumento de la esperanza de vida en LAC?.

Podemos destacar que, refiriéndose a la población norteamericana, ya se estimaba en 1991 que los adultos con necesidad de una o dos prótesis completas aumentarían de 33,6 millones a 37,9 millones en el $2020^{12}$. El envejecimiento imperativo de la población requerirá que los dentistas comprendan y atiendan estas complejas necesidades de los adultos mayores $^{13}$.

Los estudios han demostrado que el uso de las prótesis dentales sigue aumentando debido al incremento de la población envejecida. Un número elevado de personas depende de prótesis removibles para rehabilitar la función bucal normal ${ }^{14,15}$. El 
objetivo de esta revisión narrativa fue analizar la literatura científica internacional sobre las indicaciones de las sobredentaduras implantoasistidas en el paciente desdentado total.

\section{Metodología}

Se realizó un estudio retrospectivo de corte transversal mediante el análisis de la información obtenida a partir de la búsqueda de publicaciones indexadas en las bases de datos PUBMED, LILACS y EBSCO (Dentistry and Oral Sciences Source) referidos a sobredentaduras implantoasistidas en el desdentado total. La última búsqueda se realizó en diciembre 2020.

Sobredentaduras inferiores, sus limitaciones: de lo anatómico a lo socioeconómico

En 1994 Bergendal et al. ${ }^{16}$, evaluaron en 579 pacientes edéntulos las posibles localizaciones para implantes oseointegrados por delante de los segundos molares de ambos maxilares. El 78\% correspondían a los grupos de 60-70 años y viendo las diferentes alternativas clínicas se encontraron 1078 localizaciones posibles. El $100 \%$ de las mandíbulas edéntulas fueron considerados adecuadas para la colocación de implantes.

Prácticamente todas las mandíbulas edéntulas, desde lo anatómico, pueden ser sometidas a la colocación de implantes dentales, como mínimo dos en el área interforaminal (entre ambos orificios mentonianos). Esto lleva el tema de discusión hacia un nuevo enfoque, ya no si el hueso es apto volumétricamente para la colocación de implantes, sino a tener en cuenta si es conveniente o no dicho tratamiento, porque prácticamente la mandíbula es siempre apta para este tipo de intervención, aunque es válido aclarar que existen casos, los menos o las excepciones, en que no es posible colocar implantes en el maxilar inferior por razones morfológicas muy poco frecuentes, de extremísima reabsorción y hay otras razones de índole clínico-médico que pueden contraindicar una intervención quirúrgica dentomaxilar ${ }^{17}$.

Desde la perspectiva socioeconómica, se estudió la demanda subjetiva para mejorar la dentadura aplicando un método de análisis epidemiológico con un cuestionario. Se observó que un $25 \%$ de las personas se someterían incondicionalmente a tratamientos con implantes, otro $25 \%$, solo si no requería mucho tiempo, era simple y no muy costoso, siendo esta última una variable altamente significativa al momento de tomar decisiones ${ }^{18}$. Estos tratamientos pueden contribuir directamente a mejorar la salud del mayor número de pacientes posibles, y que a su vez pueden ser ejecutados por la mayor cantidad de profesionales $^{19}$.

Las sobredentaduras inferiores y la evidencia disponible como primera alternativa ideal de tratamiento en la mandíbula edéntula

Las sobredentaduras en el maxilar inferior retenidas por dos implantes colocados en la región interforaminal han sido bien documentadas en investigaciones clínicas y se han sugerido como tratamiento estándar para el paciente desdentado ${ }^{20}$. Ahora bien, es en la rehabilitación del maxilar superior edéntulo con implantes, donde se vislumbran las mayores controversias referidas a esta temática. Desde un principio, la alternativa de ferulizar implantes para aumentar supuestamente las chances de éxito a largo plazo, entendido primariamente como la mera subsistencia de los implantes en boca, influenció la planificación de los tratamientos protéticos, más allá del maxilar y el caso clínico particular.

Es comúnmente aceptado que los implantes múltiples adyacentes en pacientes desdentados deben estar necesariamente unidos, por lo que la confección de una barra que los ferulice cuando se planifica una sobredentadura surge como una opción, más que obvia y necesaria ante tal preconcepto. Al respecto, es importante destacar que la evidencia es contundente al demostrar que no existe diferencia en el éxito a largo plazo de los implantes individuales frente a los ferulizados cuando se realizan sobredentaduras en maxilar inferior, aunque si en el superior ${ }^{18,21}$.

Una sobredentadura en el maxilar inferior tiene, a 16 años de control la mitad de fracasos que una prótesis fija sobre implantes en el maxilar superior (Tabla I). Por ende, si se considera correcto o posible realizar algún tipo de prótesis implantoasistida en el maxilar superior, es necesario recomendar una sobredentadura inferior ya que tendrá el doble de probabilidades de éxito que aquellos tratamientos ${ }^{22}$. 
La atrofia ósea de la mandíbula suele ser 4 veces mayor que la del maxilar superior, por lo que la planificación del tratamiento del desdentado total debe tener siempre presente las limitaciones anatómicas del maxilar inferior en franca desventaja frente al superior, y sin dejar de considerar los efectos adversos que tendría la situación clínica en que el maxilar superior permanezca dentado o implanto restaurado frente a la mandíbula edéntula, que empeora el cuadro clínico del edentulismo inferior ${ }^{10,14,23}$.

La evidencia científica sugiere que el tratamiento de un desdentado total con una prótesis completa convencional no es el tratamiento más adecuado. Existe suficiente evidencia científica para afirmar que una sobredentadura soportada por dos implantes debiera ser la primer elección de tratamiento en un desdentado total ${ }^{24}$. Esta propuesta quedo plasmada en el consenso mundial de protesistas realizado en Montreal Canadá en el año 2002, donde los especialistas asistentes al mismo llegaron a la conclusión que: la primera opción de tratamiento que debiera recomendarse a pacientes totalmente edéntulos, ya no debería ser la confección de dos PTR convencionales, sino una sobredentadura inferior sobre dos implantes, antagonizando con una prótesis completa convencional superior ${ }^{25}$.

Tabla I. Comparativo de fracasos de los implantes, entre Prótesis Fija y Sobredentaduras en el maxilar y la mandíbula ${ }^{22}$.

\begin{tabular}{|c|c|c|}
\hline & Total Implantes & (\%) Fracasos \\
\hline Maxila Fija & 4392 & $431(9.8 \%)$ \\
\hline Maxila Sobredentadura & 930 & $198(21.3 \%)$ \\
\hline Mandíbula Fija & 8798 & 239 (2.7\%) \\
\hline Mandíbula Sobredentadura & 2383 & $118(5.0 \%)$ \\
\hline
\end{tabular}

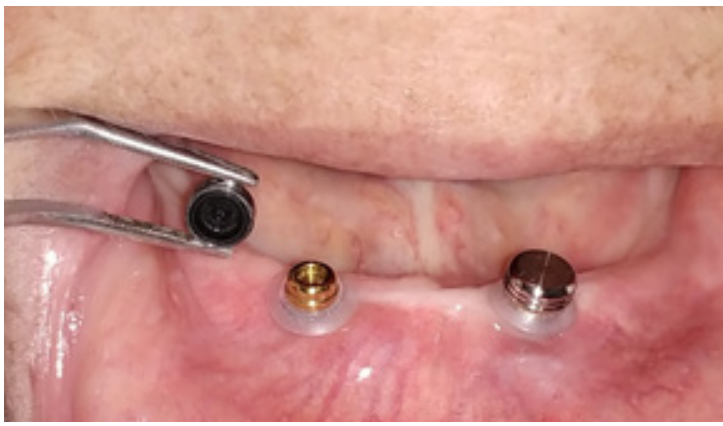

Figura 2. Maxilar inferior con dos implantes y broches independientes.
Las investigaciones clínicas en pacientes edéntulos, portadores de una sobredentadura inferior, realizadas a través de los años, han aportado numerosa evidencia. En 1987 Van Steenberghe et al. ${ }^{26}$, hicieron el primer estudio clínico prospectivo, reportando un 98\% de éxito (conservación de los implantes) con un seguimiento de 52 meses. En 1994 Mericske-Stern et al. ${ }^{27}$, publicaron en otro estudio clínico longitudinal, $97 \%$ de éxito a 5 años independientemente de si los implantes estaban individualizados o no, de si presentaban tejido queratinizado o no y del tiempo de edentulismo. En 1996 Jent et al. ${ }^{28}$, obtuvieron en un estudio clínico prospectivo multicéntrico, un $100 \%$ de éxito, pero además determinaron que la reabsorción ósea alrededor de los implantes fue de 0,5 $\mathrm{mm}$, en los primeros 5 años. En 1999 Naert et al. ${ }^{29}$, verificaron en un estudio clínico prospectivo y randomizado, un $100 \%$ de éxito a 5 años independientemente del sistema de anclaje.

Actualmente el paradigma de atención del desdentado total ${ }^{30}$, debería orientarse a la realización de sobredentaduras implantoretenidas por dos ataches a bola sobre dos implantes en el maxilar inferior (Figuras 1, 2 y 3), antagonizando con una prótesis completa convencional en el maxilar superior.

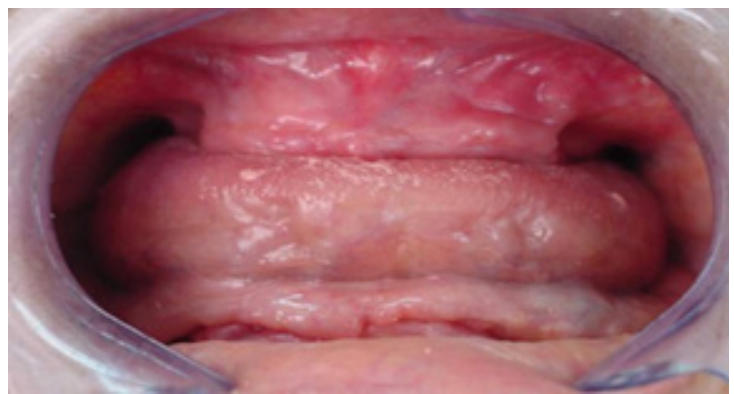

Figura 1. Paciente edéntulo.

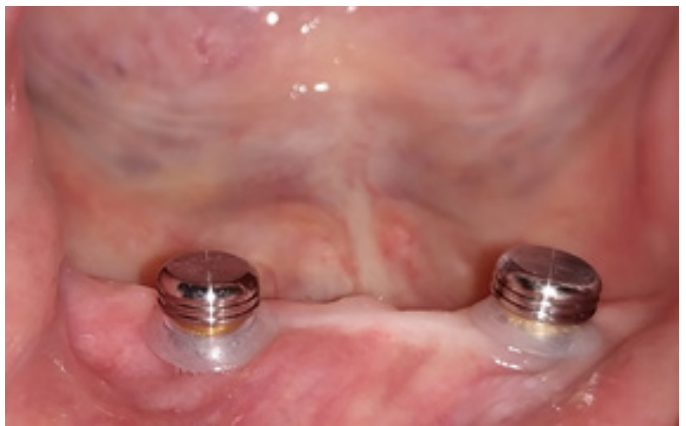

Figura 3. Broches locator para sobredentadura inferior. 


\section{Conclusiones}

En definitiva, las sobredentaduras inferiores retenidas por dos implantes han demostrado irrefutablemente ser un tratamiento de primera elección en el paciente desdentado total cuando los recursos económicos del paciente lo permiten. Esta consideración debiera orientar la capacitación profesional en el área de la prostodoncia, poniendo el foco en este creciente y cada vez más demandante grupo de pacientes.

La planificación del tratamiento protético es un complejo proceso que requiere de una combinación de información diagnóstica, expectativas del paciente, evidencia científica basada en datos propios y recopilados y una profunda y exhaustiva revisión de las alternativas de tratamiento. Con habilidades apropiadas y, hoy en día, la evidencia basada en la práctica odontológica, es un poderoso medio para establecer la efectividad de decisiones en el tratamiento de un paciente y así adquirir conocimientos clínicos y desarrollar destrezas, que nos permitan aplicarlos, en el curso de nuestra carrera profesional.

La intención de este trabajo fue difundir entre los profesionales odontólogos estos conceptos de tratamientos simples en el desdentado total, que mejoran ostensiblemente la calidad de vida de la población afectada con esta situación clínica, sin desconocer otros protocolos más complejos. El abanico de posibilidades protéticas que se abre a partir de la colocación de implantes en los maxilares edéntulos son numerosas y debiera el odontólogo conocerlas en su totalidad y al mismo tiempo debiera también desarrollar las destrezas necesarias para aplicarlos. Pero inclinarse por las alternativas más simples y probadas, así como más accesibles redundará en una mejora de la calidad de vida de los pacientes en las comunidades donde se ejerza nuestra profesión.

\section{Referencias Bibliográficas}

1. Kossioni A, McKenna G, Müller F, Schimmel M, Vanobbergen J. Higher education in Gerodontology in European Universities. BMC Oral Health. 2017;17(1):71. doi: 10.1186/s12903-017-0362-9

2. Al-Rafee MA. The epidemiology of edentulism and the associated factors: A literature Review. J Family Med Prim Care. 2020;9(4):1841-3. doi: 10.4103/jfmpc. jfmpc_1181_19

3. Tyrovolas S, Koyanagi A, Panagiotakos DB, Haro JM, Kassebaum NJ, Chrepa V et al. Population prevalence of edentulism and its association with depression and self-rated health. Sci Rep. 2016;6:37083. doi: 10.1038/ srep37083

4. Polzer I, Schimmel M, Müller F, Biffar R. Edentulism as part of the general health problems of elderly adults. Int Dent J. 2010;60(3):143-55.

5. Centro Latinoamericano de Demografía (CELADE-CEPAL). América Latina y el Caribe: estimaciones y proyecciones de población 1950-2050. Santiago de Chile: CELADE-CEPAL; 2004 (Boletín Demográfico 73). Disponible en: https://www.cepal.org/es/publicaciones/39532-america-latina-caribe-estimaciones-proyecciones-poblacion-1950-2050-latin

6. Braine T. More oral health care needed for ageing populations. Bulletin of the World Health Organization. 2005; 83 (9): 646 - 647. World Health Organization. Available at: https://apps.who.int/iris/handle/10665/269481

7. Mateos-Nozal J, Cruz-Jentofta AJ, Ribera Casado JM. Enseñanza de la Geriatria en el pregrado ¿ Siguen las Facultades de Medicina españolas las recomendaciones europeas?. Rev Esp Geriatr Gerontol. 2015;50(2):71-3. doi: 10.1016/j.regg.2014.11.007

8. Bissett S, Preshaw P. Guide to providing mouth care for older people. Nurs Older People. 2011; 23(10):14-21. doi: 10.7748/nop2011.12.23.10.14.c8837

9. Gattini C, Ruiz P. Health in South America 2012 Edition: Health Situation, Policies and Systems Overview. Pan American Health Organization, World Health Organization; 2012. Available at: https://iris.paho.org/bitstream/ handle/10665.2/28327/9789275317143_spa.pdf?sequence $=1 \&$ isAllowed $=y$

10. Rosero-Bixby L. Generational transfers and population aging in Latin America. Popul Dev Rev. 2011;37(Suppl 1):143-57. doi: 10.1111/j.1728-4457.2011.00381.x

11. United Nations, Department of Economic and Social Affairs, Population Division (2017). World Population Prospects: The 2017 Revision, Key Findings and Advance Tables. Working Paper No. ESA/P/WP/248. Available 


\section{Revista de la Facultad de Odontología}

Sobredentaduras implantoasistidas en el paciente desdentado total: bases para su indicación.
REFO

VOL. XIV | N² 2| AÑO 2021

ISSN 1668-7280

ISSN-E 2683-7986 at: https://population.un.org/wpp/publications/files/ wpp2017_keyfindings.pdf

12. Seals RR Jr, Jones JD. Evidence-based practice in removable prosthodontics. Tex Dent J. 2003;120(12):113845.

13. Ettinger RL, Goettsche ZS, Qian F. Curriculum content in geriatric dentistry in USA dental schools. Gerodontology. 2018;35(1):11-17. doi: 10.1111/ger.12305

14. Emami E, de Souza RF, Kabawat M, Feine JS. The impact of edentulism on oral and general health. Int J Dent. 2013;2013:498305. doi: 10.1155/2013/498305

15. Douglass CW, Shih A, Ostry L. Will there be a need for complete dentures in the United States in 2020? J Prosthet Dent. 2002;87(1):5-8. doi:10.1067/mpr.2002.121203

16. Bergendal T, Hugoson A, Kvint S, Lundgren D. A radiological inventory of possible sites for cylinder implants in edentulous regions of the jaws. An epidemiological study. Swed Dent J. 1994;18(3):75-85.

17. Schwartz IS, Morrow RM. Overdentures. Principles and procedures. Dent Clin North Am. 1996;40(1):169-94. 18. Sadowsky SJ. Mandibular implant-retained overdentures: a literature review. J Prosthet Dent. 2001;86(5):46873. doi: 10.1067/mpr.2001.119921

19. Taylor TD, Wiens J, Carr A. Evidence-based considerations for removable prosthodontic and dental implant occlusion: a literature review. J Prosthet Dent. 2005;94(6):555-60. doi: 10.1016/j.prosdent.2005.10.012 20. Ali SM, El Talawy DB. Locator versus stress free implant bar attachments for two-implants immediately loaded by mandibular overdentures. A one year randomized study. Egypt Dent J. 2021;67(2):1345-46. doi: 10.21608/EDJ.2021.55094.1425

21. Alqutaibi AY, Elawady DMA. Implant splinting in mandibular overdentures: a systematic review with meta-analysis of randomized clinical trials. Quintessence Int. 2020;51(4):294-302. doi: 10.3290/j.qi.a44144

22. Goodacre CJ, Kan JY, Rungcharassaeng K. Clinical complications of osseointegrated implants. J Prosthet Dent. 1999;81(5):537-52. doi: 10.1016/s00223913(99)70208-8
23. Tallgren A. The continuing reduction of the residual alveolar ridges in complete denture wearers: a mixed-longitudinal study covering 25 years. 1972. J Prosthet Dent. 2003;89(5):427-35. doi: 10.1016/s00223913(03)00158-6

24. Thomason JM. The McGill Consensus Statement on Overdentures. Mandibular 2-implant overdentures as first choice standard of care for edentulous patients. Eur J Prosthodont Restor Dent. 2002;10(3):95-6.

25. Feine JS, Carlsson GE, Awad MA, Chehade A, Duncan WJ, Gizani S et al. The McGill consensus statement on overdentures. Mandibular two-implant overdentures as first choice standard of care for edentulous patients. Montreal, Quebec, May 24-25, 2002. Int J Oral Maxillofac Implants. 2002;17(4):601-2.

26. Van Steenberghe D, Quirynen M, Calberson L, Demanet M. A prospective evaluation of the fate of 697 consecutive intra-oral fixtures ad modum Branemark in the rehabilitation of edentulism. J Head Neck Pathol. 1987;6:53-8.

27. Mericske-Stern R, Steinlin Schaffner T, Marti P, Geering $\mathrm{AH}$. Peri-implant mucosal aspects of ITI implants supporting overdentures. A five-year lon- gitudinal study. Clin Oral Implants Res. 1994;5(1):9-18. doi: 10.1034/j.16000501.1994.050102.x

28. Jemt T, Chai J, Harnett J, Heath MR, Hutton JE, Johns $\mathrm{RB}$, et al. A 5-year prospective multicenter follow-up report on overdentures supported by osseointegrated implants. Int J Oral Maxillofac Implants.1996;11(3):291-8.

29. Naert I, Gizani S, Vuylsteke M, van Steenberghe D. A 5-year prospective randomized clinical trial on the influence of splinted and unsplinted oral implants retaining a mandibular overdenture: prosthetic aspects and patient satisfaction. J Oral Rehabil 1999;26(3):195-202. doi: 10.1046/j.1365-2842.1999.00369.x

30. Thomason JM, Kelly SA, Bendkowski A, Ellis JS. Two implant retained overdentures--a review of the literature supporting the McGill and York consensus statements. J Dent. 2012;40(1):22-34. doi: 10.1016/j. jdent.2011.08.017 